\title{
Ações de prevenção primária e secundária relacionadas aos fatores de risco para osteoporose
}

\section{Primary and secondary prevention actions related to risk factors for osteoporosis \\ Acciones de prevención primaria y secundaria relacionadas con los factores de riesgo para osteoporosis}

\author{
Giulia Ohana Franco iD \\ Faculdade Ceres - FACERES - São José do Rio Preto (SP) - Brasil \\ Giulia Sestini (iD \\ Faculdade Ceres - FACERES - São José do Rio Preto (SP) - Brasil \\ Gabriel Antonio Cabriott Dumbra iD \\ Faculdade Ceres - FACERES - São José do Rio Preto (SP) - Brasil \\ Mariana Storino Conte (iD \\ Faculdade Ceres - FACERES - São José do Rio Preto (SP) - Brasil \\ Felipe Colombelli Pacca (iD \\ Faculdade Ceres - FACERES - São José do Rio Preto (SP) - Brasil \\ Daniela Vichiato Polizelli Roma (iD \\ Faculdade Ceres - FACERES - São José do Rio Preto (SP) - Brasil \\ Patrícia da Silva Fucuta (iD \\ Faculdade Ceres - FACERES - São José do Rio Preto (SP) - Brasil \\ Tamara Veiga Faria iD \\ Faculdade Ceres - FACERES - São José do Rio Preto (SP) - Brasil
}

\section{RESUMO}

Objetivo: Analisar a efetividade do teste da International Osteoporosis Foundation (IOF) para as prevenções primária e secundária relacionadas aos fatores de risco para a osteoporose. Métodos: Estudo transversal realizado no interior de São Paulo, Brasil, durante a Campanha de Prevenção à Osteoporose realizada em outubro de 2016. Participaram 400 pessoas, selecionadas aleatoriamente, entrevistadas de acordo com o teste de um minuto para risco de osteoporose da IOF. A análise estatística utilizou o teste de Kolmogorov-Smirnov, o teste qui-quadrado de Pearson, o Mann-Whitney e a análise multivariada para fatores de risco associados à osteoporose pelo modelo de regressão logística binária. Os resultados foram apresentados em odds ratio, com intervalo de confiança de 95\%. Resultados: A amostra foi composta por 260 mulheres e 140 homens, com mediana de 57 anos, e $95 \%$ indicaram possuir algum fator de risco. As questões com maior índice de positividade indicaram que ambos os sexos estão expostos à baixa exposição ao sol, à baixa ingesta de alimentos ricos em vitamina $D(p=0,140)$ e ao hábito de atividades físicas por tempo inferior a $30 \mathrm{~min}(p=0,657)$. O índice de massa corporal (IMC) menor que $19 \mathrm{~kg} / \mathrm{m} 2(p=0,336)$ indicou menor positividade. A regressão logística mostrou associação entre quatro fatores de risco (densitometria óssea, queda por fraqueza, mudança de altura após os 40 anos e sexo) e a população em estudo acima de 60 anos. Conclusão: O teste da IOF se mostrou uma ferramenta funcional na promoção da saúde e atenção primária, podendo trazer benefícios socioeconômicos.

Descritores: Prevenção Primária; Prevenção Secundária; Fatores de Risco; Osteoporose; Saúde Pública.

\section{ABSTRACT}

Objective: To analyze the effectiveness of the International Osteoporosis Foundation (IOF) test for primary and secondary prevention related to risk factors for osteoporosis. Methods: This is a cross-sectional study conducted in the interior of São Paulo, Brazil, during the Osteoporosis Prevention Campaign carried out in October 2016. 400 people, selected at random, interviewed according to the IOF one-minute osteoporosis risk test, participated. Statistical analysis used the Kolmogorov-Smirnov test,

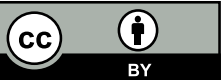


Pearson's chi-square test, Mann-Whitney, and multivariate analysis for risk factors associated with osteoporosis using the binary logistic regression model. The results were presented in odds ratios, with a 95\% confidence interval. Results: The sample consisted of 260 women and 140 men, with a median of 57 years, and 95\% indicated having some risk factor. The questions with the highest positivity index indicated that both sexes are exposed to low exposure to the sun, low intake of foods rich in vitamin $D(p=0.140)$, and the habit of physical activities for less than $30 \mathrm{~min}(p=0.657)$. The body mass index (BMI) less than $19 \mathrm{~kg} / \mathrm{m} 2$ $(p=0.336)$ indicated less positivity. Logistic regression showed an association between four risk factors (bone densitometry, fall due to weakness, change in height after 40 years and sex) and the study population over 60 years. Conclusion: The IOF test proved to be a functional tool in promoting health and primary care, and can bring socioeconomic benefits.

Descriptors: Primary Prevention; Secondary Prevention; Risk Factors; Osteoporosis; Public Health.

\section{RESUMEN}

Objetivo: Analizar la efectividad de la prueba de la International Osteoporosis Foundation (IOF) para las prevenciones primaria y secundaria relacionadas con los factores de riesgo para osteoporosis. Métodos: Estudio transversal realizado en una ciudad de São Paulo, Brasil, durante la Campaña de Prevención de Osteoporosis realizada en octubre de 2016. Participaron 400 personas que han sido elegidas de modo aleatorio y entrevistadas según la prueba de un minuto para riesgo de osteoporosis de la IOF. El análisis estadístico utilizó la prueba de Kolmogorov-Smirnov, la prueba de Chi-cuadrado de Pearson, la de Mann-Whitney y el análisis multivariado para los factores de riesgo asociados con la osteoporosis por el modelo de regresión logística binaria. Se ha presentado los resultados en odds ratio con intervalo de confianza del 95\%. Resultados: La muestra fue de 260 mujeres y 140 hombres con mediana de la edad de 57 años y el 95\% indicaron tener algún factor de riesgo. Las preguntas con mayor índice de positividad han indicado que ambos sexos tienen baja exposición solar, baja ingesta de alimentos con vitamina $D(p=0,140)$ y la costumbre de actividades físicas de tiempo menor que $30 \mathrm{~min}(p=0,657)$. El índice de masa corporal (IMC) de menos de $19 \mathrm{~kg} / \mathrm{m}^{2}(p=0,336)$ ha indicado menor positividad. La regresión logística ha mostrado asociación entre cuatro factores de riesgo (densitometría ósea, caída causada por debilidad muscular, cambio de altura después de los 40 años y sexo) y la población del estudio con más de 60 años. Conclusión: La prueba de la IOF se presentó como una herramienta funcional para la promoción de la salud y atención primaria lo que puede llevar a beneficios socioeconómicos.

Descriptores: Prevención Primaria; Prevención Secundaria; Factores de Riesgo; Osteoporosis; Salud Pública.

\section{INTRODUÇÃO}

Um dos grandes desafios a ser considerado no Brasil e na saúde coletiva é o envelhecimento da população, tornando as prevenções primária e secundária de doenças crônicas indispensáveis, principalmente quando se consideram as causas de morbidade e mortalidade entre adultos e idosos ${ }^{(1)}$.

É importante destacar que o país apresenta uma população heterogênea com relação a questões demográficas, culturais, raciais, sociais e no acesso a serviços de saúde, fator que dificulta estudos epidemiológicos sobre a prevalência da osteoporose e sua interferência nos gastos com a saúde pública(2).

Sobre a osteoporose, ressalta-se que é uma doença silenciosa cuja prevalência, de acordo com os estudos nacionais, atinge de $15 \%$ a $33 \%$ das mulheres pós-menopausadas ${ }^{(3-5)}$. Outros dados indicam que atinge 10 milhões de brasileiros, tendo a maioria mais de 65 anos. Quando comparada a outras doenças, em mulheres acima de 45 anos, a osteoporose apresenta um número maior de dias de internação para tratamento, gerando maior gasto financeiro no âmbito da saúde pública(2).

A maioria dos artigos científicos sobre osteoporose trata de estudos sobre fraturas ósseas consequentes da evolução e de complicações da doença, as quais representam altos custos à saúde pública e têm relação direta com o impacto negativo na qualidade de vida da população idosa, além de representar uma elevada taxa de mortalidade ${ }^{(2,4)}$. Desse modo, a indicação de tratamento para prevenção de fraturas ósseas relacionadas à osteoporose significa um impacto no sistema de saúde pública ${ }^{(5,6)}$, justificando, assim, a necessidade tanto da prevenção primária quanto secundária ${ }^{(6)}$.

O maior interesse clínico deve ser a diminuição da incidência da osteoporose e suas complicações, com foco nos fatores de risco modificáveis, como a exposição solar inadequada, a não suplementação de vitamina $D$ e o tabagismo ${ }^{(5,7)}$.

Mais recentemente, as recomendações de saúde pública, tais como o hábito de evitar a exposição solar, destinam-se à prevenção do câncer de pele, o que pode contribuir para ambos os sexos adquirirem mais um fator de risco para osteoporose ${ }^{(2,8)}$. Entende-se que, no contexto da prevenção primária, estão incluídas medidas de incentivo à prática de exercícios físicos, hábitos alimentares saudáveis, ingestão adequada de cálcio e vitamina $\mathrm{D}$ na dieta, 
bem como a exposição ao sol. Na comparação entre os sexos, nota-se que, em mulheres na pósmenopausa, as ações de prevenção primária também auxiliam na diminuição do ritmo de perda óssea ${ }^{(7)}$.

A prevenção secundária, por outro lado, englobaria estratégias populacionais para sua detecção precoce, incluindo rastreamento de populações-alvo. Os métodos de rastreamento direcionam-se mais especificamente para a saúde da mulher, principalmente durante a pós-menopausa, enquanto a cobertura ao sexo masculino não possui a mesma abordagem ${ }^{(7)}$.

Estudos europeus, norte-americanos e asiáticos, visando prevenir as complicações da osteoporose, mostraram a relevância clínica de ferramentas e estratégias que possam contribuir para a identificação de indivíduos com maior risco de desenvolver osteoporose e, consequentemente, colaborar para a promoção da saúde ${ }^{(9)}$.

Nesse cenário, para atender às necessidades da saúde pública, a fim de identificar ações modificáveis e ter um diagnóstico precoce, estudos têm mostrado a importância clínica de ferramentas capazes de indicar precocemente fatores de risco como principal meta na introdução de estratégias efetivas de prevenção da osteoporose, as quais poderão ser usadas para diferenciar indivíduos com maior risco de desenvolvê-la ${ }^{(2,7,8)}$.

Contudo, mesmo que haja tratamentos oferecidos pelo SUS capazes de colaborar para a prevenção dessa doença, a falta do conhecimento epidemiológico adequado ocasiona o pouco acesso da população brasileira ao diagnóstico precoce e prejuízos aos programas de saúde. Ademais, as estratégias de prevenção da osteoporose têm como principal meta a identificação precoce dos fatores de risco $^{(3)}$. Logo, foi proposto o desenvolvimento do presente estudo, que tem como objetivo analisar a efetividade do teste da International Osteoporosis Foundation (IOF) para as prevenções primária e secundária relacionadas aos fatores de risco para a osteoporose.

\section{MÉTODOS}

Trata-se de um estudo transversal desenvolvido em São José do Rio Preto, São Paulo, Brasil, local onde se realizou uma campanha de prevenção da osteoporose promovida por uma faculdade. Durante os dias 23 e 24 de outubro de 2016, em horário comercial, em um shopping localizado na região central da cidade, com o apoio de 44 estudantes, nutricionistas, fisioterapeutas e reumatologistas, foram realizadas as orientações para prevenção da osteoporose. Esse evento teve ampla divulgação em redes sociais e mídias visuais da região e contou com o apoio da IOF.

Para o delineamento dos fatores de risco relacionados à osteoporose e a caracterização da população, aplicou-se o teste de um minuto para risco de osteoporose da IOF com 400 participantes aleatoriamente selecionados durante o evento. Incluíram-se no estudo pessoas maiores de 18 anos dispostas a receber orientações sobre a doença e atendidas pela atenção primária do município de origem, sendo excluídos os participantes que responderam aos questionários de forma incompleta.

Restringiu-se a coleta de dados ao teste da IOF, formado por 19 questões, cujas respostas (afirmativas ou negativas) levavam cerca de um minuto, representando um método de triagem aleatório de fatores de risco, sem restrição de idade e sexo. Suas afirmativas não significam diagnóstico de osteoporose, mas apontam fatores de risco clinicamente comprovados de desenvolver osteoporose e de fratura ${ }^{(10-12)}$.

A partir dos resultados obtidos no teste, a sugestão da IOF é que sejam realizadas ações de esclarecimento e informação à população sobre os riscos aos quais estão sujeitos, mediante planos de prevenção, mesmo que haja somente respostas negativas ao questionário. Há estudos internacionais que também já utilizaram esse teste como ferramenta de conscientização, alertando sobre possíveis fatores de risco para a osteoporose e, consequentemente, a importância de sua detecção precoce ${ }^{(10-12)}$.

Com base no teste, as variáveis do estudo foram: diagnóstico de osteoporose, realização de densitometria óssea, tratamento anterior e atual, histórico familiar, presença de cifose, idade, etilismo, tabagismo, história de fratura por queda, queda por fraqueza, mudança de altura após os 40 anos, índice de massa corpórea, uso de corticoterapia por três meses, comorbidades (como artrite reumatoide e doença da tireoide), exercícios < 30 min diários, exposição solar e ingesta de vitamina $\mathrm{D}$. A análise exploratória dos dados incluiu média, mediana, desvio-padrão e variação para variáveis contínuas, bem como número e proporção para variáveis categóricas. A distribuição normal das variáveis contínuas foi analisada pela assimetria, curtose e teste de Kolmogorov-Smirnov. Realizaram-se associações das variáveis categóricas entre dois grupos pelo teste qui-quadrado de Pearson e das variáveis contínuas pelo teste de Mann-Whitney. Realizou-se a análise multivariada para fatores de risco associados à osteoporose pelo modelo de regressão logística binária (método stepwise with backward elimination), apresentando-se os resultados em odds ratio com intervalo de confiança de $95 \%$. Além disso, com auxílio do software IBM-SPSS Statistics, versão 24 (IBM Corporation, NY, USA), realizou-se a análise estatística. Lembrando que todos os testes foram bicaudais, considerando-se valores de $p<0,05$ significantes. 
Este estudo obteve a aprovação do Comitê de Ética em Pesquisa (CEP), com o Parecer n. ${ }^{\circ} 1.445 .235$, de acordo com a Resolução CNS n. ${ }^{\circ} 466 / 12$.

\section{RESULTADOS}

Participaram do presente estudo 260 mulheres e 140 homens (220 adultos e 180 idosos), com mediana de idade de 57 anos, sendo máximo de 82 anos e mínimo de 32 anos. Aproximadamente $95 \%$, de ambos os sexos, respondeu afirmativamente a uma questão do teste pelo menos, indicando a presença de fatores de risco clinicamente comprovados para osteoporose.

A incidência de fatores de risco por sexo mostrou que ambos seguiram a mesma proporção. Somente 12 mulheres (5\%) e 5 homens (4\%) não indicaram possuir fator de risco para osteoporose, segundo o teste da IOF. A análise dos questionários confirmou que o diagnóstico de osteoporose continua mais incidente em mulheres quando comparadas aos homens $(p<0,001)$. As mulheres também são submetidas com maior frequência $(n=116 ; 44,6 \%)$ à densitometria óssea quando comparadas aos homens $(n=23 ; 16,4 \%)(p<0,001)$.

Em relação à incidência de respostas positivas por questão, observou-se que as questões de número 3, 17, 16 e 19 obtiveram maior índice de positividade, referentes, respectivamente, aos seguintes enunciados: "você tem 40 anos ou mais?" ( $n=318 ; 79,5 \%)$, "sua cota diária de exercícios é inferior a 30 minutos?" (n=174; 43,5\%), "você fuma ou já foi fumante?" ( $n=147 ; 36,75 \%$ ) e "você fica menos de 10 minutos por dia ao ar livre com parte de seu corpo exposta ao sol ( $n=134 ; 33,5 \%)$ sem ingerir alimentos ou suplementos ricos em vitamina D?" ( $n=81 ; 20,25 \%)$ (Tabela I).

A maioria dos participantes com mais de 40 anos era composta por mulheres $(76,5 \% ; n=119 ; p=0,046)$. Em contrapartida, ambos os sexos se mostraram expostos aos seguintes fatores de risco: falta de hábito de praticar atividades físicas, a baixa/ausente ingestão de leite e derivados, e a exposição solar precária. $O$ fator de risco "tabagismo" foi equivalente estatisticamente para os sexos e o etilismo permaneceu mais prevalente nos homens (Tabela I).

A alternativa de menor positividade durante a pesquisa foi a de número 7 , que aborda a verificação do peso a partir do índice de massa corporal (IMC) menor que $19 \mathrm{~kg} / \mathrm{m}^{2}$.

Em relação à comparação entre sexo e "presença de osteoporose nos pais", houve diferença significativa $(p=0,005)$, assim como a queda por fraqueza $(p=0,04)$, mudança de altura após os 40 anos $(p=0,005)$, presença de artrite reumatoide $(p=0,007)$ e doença da tireoide $(p=0,011)$. Quanto à faixa etária, nos participantes com idade inferior a 40 anos $(20,5 \%)$, o uso de corticoides $(p=0,129)$ não foi prevalente.

Ao se controlar o efeito das variáveis em conjunto pelo modelo de regressão logística binária, observou-se uma associação entre os fatores de risco densitometria óssea, queda por fraqueza, mudança de altura após os 40 anos e sexo no que diz respeito à população em estudo acima de 60 anos, conforme descrito pela Tabela II.

Tabela I - Representa o número de respostas categorizadas em sim ou não e por questão do teste da IOF (International Osteoporosis Foundation) classificada por sexo $(n=400)$. São José do Rio Preto, São Paulo, 2016.

\begin{tabular}{|c|c|c|c|c|c|}
\hline \multirow{2}{*}{ Variáveis } & \multicolumn{2}{|c|}{ Masculino $(n=140)$} & \multicolumn{2}{|c|}{ Feminino $(n=260)$} & \multirow[b]{2}{*}{ Valor $p$} \\
\hline & Sim & Não & Sim & Não & \\
\hline Diagnóstico de osteoporose & $6(4,3 \%)$ & $134(95,7 \%)$ & $45(17,3 \%)$ & $215(82,7 \%)$ & $0,000^{*}$ \\
\hline Densitometria óssea & $23(16,4 \%)$ & $117(83,6 \%)$ & $116(44,6 \%)$ & $144(55,4 \%)$ & $<0,001^{*}$ \\
\hline Tratamento anterior de osteoporose & $6(4,3 \%)$ & $134(95,7 \%)$ & $36(13,8 \%)$ & $224(86,2 \%)$ & $0,003^{*}$ \\
\hline Tratamento atual de osteoporose & $4(2,9 \%)$ & $136(97,1 \%)$ & $27(10,4 \%)$ & $233(89,6 \%)$ & $0,007^{*}$ \\
\hline Osteoporose nos pais & $19(13,6 \%)$ & $121(86,4 \%)$ & $67(25,8 \%)$ & $193(74,2 \%)$ & $0,005^{*}$ \\
\hline Cifose pais & $16(11,4 \%)$ & $124(88,6 \%)$ & $42(16,2 \%)$ & $218(83,8 \%)$ & 0,2 \\
\hline Idade $>40$ anos & $119(85 \%)$ & $21(15 \%)$ & $199(76,5 \%)$ & $61(23,5 \%)$ & $0,046^{*}$ \\
\hline Fratura pós-queda & $35(25 \%)$ & $105(75 \%)$ & $48(18,5 \%)$ & $212(81,5 \%)$ & 0,129 \\
\hline Queda por fraqueza & $11(7,9 \%)$ & $129(92,1 \%)$ & $48(18,5 \%)$ & $212(81,5 \%)$ & $0,004^{*}$ \\
\hline Mudança de altura após os 40 anos & $24(17,1 \%)$ & $116(82,9 \%)$ & $67(25,8 \%)$ & $193(74,2 \%)$ & $0,050^{*}$ \\
\hline IMC baixo & $9(6,4 \%)$ & $131(93,6 \%)$ & $11(4,2 \%)$ & $249(95,8 \%)$ & 0,336 \\
\hline Corticoterapia por 3 meses & $17(12,2 \%)$ & $123(87,2 \%)$ & $47(18,1 \%)$ & $213(81,9 \%)$ & 0,129 \\
\hline Artrite reumatoide & $11(7,9 \%)$ & $129(92,1 \%)$ & $46(17,7 \%)$ & $214(82,3 \%)$ & $0,007^{*}$ \\
\hline Doença da tireoide & $7(5 \%)$ & $133(95 \%)$ & $34(13,1 \%)$ & $226(86,9 \%)$ & $0,011^{*}$ \\
\hline Etilismo & $18(12,9 \%)$ & $122(87,1 \%)$ & $10(3,8 \%)$ & $250(96,2 \%)$ & $0,001^{*}$ \\
\hline Tabagismo & $57(40,7 \%)$ & $83(59,3 \%)$ & $90(34,6 \%)$ & $170(65,4 \%)$ & 0,228 \\
\hline Exercícios $<30 \mathrm{~min}$ & $63(45 \%)$ & $77(55 \%)$ & $111(42,7 \%)$ & $149(57,3 \%)$ & 0,657 \\
\hline Ausência de leite e derivados & $34(24,3 \%)$ & $106(75,7 \%)$ & $47(18,1 \%)$ & $213(81,9 \%)$ & 0,141 \\
\hline Exposição solar $<10 \mathrm{~min}$ & $48(34,3 \%)$ & $92(65,7 \%)$ & $86(33,1 \%)$ & $174(66,9 \%)$ & 0,807 \\
\hline
\end{tabular}

* valores $p<0,05$, estatisticamente significantes; IMC: índice de massa corpórea; min: minutos 
Tabela II - Representação da regressão logística binária de fatores de risco para osteoporose associado ao idoso. São José do Rio Preto, São Paulo, 2016.

\begin{tabular}{lccc}
\hline Variáveis & $\boldsymbol{p}$ - valor & OR * & IC 95\%** $^{\text {* }}$ \\
\hline Densitometria óssea & $<0,001$ & 5,013 & $2,823-8,902$ \\
Queda por fraqueza & 0,027 & 2,104 & $1,087-4,074$ \\
Mudança de altura após os 40 anos & $<0,001$ & 3,787 & $2,104-6,816$ \\
Sexo & $<0,001$ & 6,086 & $3,521-10,518$ \\
\hline
\end{tabular}

*OR: Odds ratio: razão de chances para osteoporose; ${ }^{* \star} \mathrm{IC}$ 95\%: intervalo de 95\% de confiança para OR

\section{DISCUSSÃO}

O teste de um minuto para risco de osteoporose da IOF é um questionário que se apresenta como uma estratégia para a definição das ações a serem desenvolvidas durante as prevenções primária e secundária de osteoporose, pois permite identificar quais fatores de risco incidem na região analisada e sua relação com a faixa etária e o sexo. $\mathrm{Na}$ literatura, a maioria dos artigos discorre sobre as fraturas ósseas como complicações da osteoporose, os impactos do tratamento com bifosfonados no sistema de saúde pública e as diretrizes de tratamento(5,6,13).

Os resultados do presente estudo demonstram que o teste da IOF é capaz de estimular o seu uso pelos profissionais na promoção de saúde, pois é de fácil acesso, baixo custo, rápido e pode ser utilizado como ferramenta para os grupos de orientação da atenção primária, além de colaborar para a caracterização da população das redes primárias. Nesse mesmo sentido, permite identificar que, além de apresentar uma maior incidência na população feminina, conforme descrito por outros estudos ${ }^{(14,15)}$, deve-se considerar outros fatores de risco de maior significância, como a falta de hábito de praticar atividades físicas, a baixa/ausente ingestão de leite e derivados, e a exposição solar precária.

A história familiar foi vista como fator de risco para osteoporose, a qual está mais presente nas mulheres. Para entender esse achado podem-se considerar duas hipóteses já destacadas na literatura científica: a genética determina o pico de massa óssea e, no caso das mulheres pós-menopausa, a taxa de perda óssea nos primeiros anos após a menopausa (também geneticamente determinada) ${ }^{(14,15)}$. E a segunda hipótese: as mulheres continuam sendo referência familiar como cuidadoras e conhecedoras de sua saúde e de seus familiares próximos, como filhos e esposos ${ }^{(16)}$, corroborando com os dados do presente estudo, no qual as mulheres, além de terem mais lembranças sobre seus históricos familiares, têm maior acesso ao sistema de saúde e participam de campanhas de prevenção de doença.

O presente estudo evidencia que a densitometria óssea é mais realizada pelas mulheres, o que favorece a identificação de baixa densidade mineral óssea (DMO) como fator de risco para a osteoporose nesse grupo. A indicação do exame deve considerar a faixa etária de risco correspondente: mulheres no climatério e pós-menopausa, histórico de fratura óssea após os 50 anos, anormalidades vertebrais radiológicas e adultos que apresentem condições associadas à baixa massa óssea ${ }^{(5,7,13)}$.

Em relação ao hipotireoidismo, os dados ressaltam a importância do rastreamento dessa doença na população e sua forte relação como fator de risco para a osteoporose, principalmente na população feminina. As alterações hormonais consequentes dessa patologia podem interferir no ciclo menstrual e nos níveis de estrogênio, relacionando-se ao desenvolvimento da osteoporose por perda da densidade mineral óssea (DMO) provocada pelo aumento da reabsorção pelos osteoclastos ${ }^{(5,15,16-19)}$.

O presente estudo demonstra que a maioria dos homens e das mulheres não fazem ingestão adequada de leite e derivados. Esses dados contrariam outros estudos brasileiros, nos quais apenas $15,8 \%$ dos participantes evitavam leite e derivados ${ }^{(5,18)}$. Há, ainda, estudos indicando maior incidência da ausência de leite e derivados na população masculina, a qual tem preferência por ovos e carnes ${ }^{(20,21)}$.

Dessa forma, a baixa ingesta de leite e derivados como fator de risco para a osteoporose pode se tornar ainda mais presente, pois já é relatado que a ingesta é menor entre indivíduos mais jovens, que devem ser incentivados ao consumo de leite e derivados ${ }^{(21)}$. Uma provável explicação para tal diferença pode estar em motivos econômicos e socioculturais relacionados a dietas com ausência de lactose, cada vez mais frequentes nas populações de regiões tropicais, nas quais outros alimentos ricos em cálcio não são considerados ${ }^{(20,21)}$.

Assim como outros estudos brasileiros e italianos, que descrevem o tempo inadequado de atividade física como fator de risco incidente na população estudada, a prática de exercícios físicos regulares não se apresenta como rotina para ambos os $\operatorname{sexos}^{(10-12,15,22,23)}$. 
Apesar de os participantes do estudo viverem em uma região tropical, a exposição diária ao sol não é uma realidade, o que pode estar relacionado ao baixo conhecimento dos benefícios da exposição solar em horários adequados por parte da população leiga. Além disso, considerando-se o padrão de vida moderna e a violência, veículos motorizados são cada vez mais utilizados para locomoção do que caminhadas, havendo a preferência pela realização de exercícios físicos em academias fechadas (não ao ar livre) e pelo uso de veículos motorizados para locomoção, o que propicia a falta de exposição solar diária ${ }^{(11,23)}$.

Outras questões que poderiam explicar esse decréscimo na exposição solar seriam as campanhas que propõem ações para amenizar os fatores de risco do câncer de pele e o aumento da temperatura nas cidades de clima tropical. Assim, a reposição de vitamina $\mathrm{D}$ e de cálcio para a população com fatores de risco poderia ser uma opção na saúde pública(7,18).

A relação entre a exposição solar e a prevenção da osteoporose pode ser explicada pela vitamina $\mathrm{D}$, nome popular para o hormônio esteroide calcitriol, que auxilia na absorção de cálcio e no metabolismo ósseo. Essa vitamina é formada por duas formas bioequivalentes, sendo a vitamina D2 (ergocalciferol) obtida pelo organismo através dos vegetais e da suplementação, e a vitamina D3 (colecalciferol) obtida, principalmente, pela exposição da pele à luz solar e pela ingestão de certos alimentos, como leite e derivados ${ }^{(24,25)}$.

Outro fator de risco identificado na população em estudo é a prevalência da artrite reumatoide no sexo feminino quando comparada ao sexo masculino. De fato, é descrito que a osteoporose pode ser uma complicação da artrite reumatoide e de outras doenças autoimunes, entretanto a literatura ainda é controversa ao se considerar a relação com essas doenças. Uma das possíveis explicações para tal relação estaria em falhas nos sistemas de regulação óssea. É conhecido que pacientes com artrite reumatoide têm maior risco de fraturas, apresentam uma menor DMO no quadril e na coluna vertebral, e têm maior risco de osteoporose no caso de pacientes idosos. Além disso, outro fator que colabora para a relação das doenças reumáticas e a osteoporose é o uso prolongado de corticoides como tratamento ${ }^{(7,19,25)}$. Os glicocorticoides reduzem a quantidade e a função dos osteoblastos, a expressão do RANK-L é incrementada e a OPG é reduzida, favorecendo a ativação dos osteoclastos e, consequentemente, contribuindo para a baixa $\mathrm{DMO}^{(25)}$.

De acordo com um estudo, o tabaco poderia ter uma ação inibidora dos osteoblastos, mas, por outro lado, a relação do tabagismo com a baixa DMO também poderia ser esclarecida por interferências na absorção do cálcio e menor nível de estradiol ${ }^{(15)}$. No atual estudo não se pôde entender essa relação, entretanto seus dados estão de acordo com estudos europeus que descrevem que as mulheres estão adquirindo mais dois agravantes a favor da osteoporose - tabagismo e etilismo -, uma vez que esses hábitos estão cada vez mais frequentes na população feminina ${ }^{(5,10-12)}$.

É conhecida a associação da idade avançada com a osteoporose, assim como a maior chance de desenvolver essa doença em mulheres pós-menopausa pela redução da $\mathrm{DMO}^{(15,24)}$. Na presente pesquisa, através da regressão logística, o envelhecimento foi relacionado ao aumento de chances de desenvolver a osteoporose por queda por fraqueza, mudança de estatura após os 40 anos e densitometria óssea.

O teste da IOF também conseguiu identificar a prevalência, em ambos os sexos, de IMC maior que 19, o que pode retratar o predomínio da população obesa. Portanto, são dados semelhantes à literatura e esclarecem que a população estudada teria um fator protetor sobre a massa óssea relacionada à maior produção de estrógenos consequentes da ação da aromatase nos adipócitos ${ }^{(15,23)}$.

Como limitação do estudo tem-se a falta de dados sociodemográficos para relacionar os fatores de risco associados à doença, um ponto a ser observado. Entretanto a implementação do teste da IOF na saúde coletiva pode trazer melhorias para a prevenção dessa doença no país, permitindo a diminuição do impacto socioeconômico nos serviços de saúde.

Cabe salientar que, entre os fatores de risco identificados pelo teste, aqueles relacionados à suplementação de cálcio e vitamina $\mathrm{D}$, bem como à exposição solar, representam ações imediatas que promovem a saúde da população em ambos os sexos.

Por fim, fatores como realização de densitometria óssea, mudança de altura e fratura representam sinais de alerta aos profissionais da saúde, pois aumentam a chance de mulheres idosas desenvolverem osteoporose.

\section{CONCLUSÃO}

A análise do teste da IOF, desenvolvida com relação a sua efetividade na identificação de fatores de risco, demonstrou tratar-se de um teste funcional e confiável, indicando-o como ferramenta na promoção de saúde e 
atenção primária. Com base nos resultados, cabe ao profissional de saúde pública orientar os usuários do sistema de saúde sobre as medidas preventivas a serem seguidas, visando diminuir as complicações dessa doença, como as fraturas ósseas, reduzindo custos, incapacidades e mortes.

\section{CONFLITOS DE INTERESSE}

Os autores declaram a inexistência de conflitos de interesses, tanto na execução das ações do projeto de pesquisa como na escrita deste manuscrito.

\section{COLABORAÇÕES}

International Osteoporosis Foundation (IOF).

\section{CONTRIBUIÇÕES}

Giulia Franco contribuiu com o delineamento e elaboração da pesquisa e a análise dos dados. Giulia Sestini, Gabriel Antonio Cabriott Dumbra e Mariana Storino Conte contribuíram com o delineamento e elaboração da pesquisa, e a coleta e análise dos dados. Felipe Colombelli Pacca contribuiu com o delineamento e elaboração da pesquisa. Daniela Vichiato Polizelli Roma contribuiu com a coleta dos dados e elaboração da pesquisa. Patrícia da Silva Fucuta contribuiu com a análise dos dados e a revisão do artigo. Tamara Veiga Faria contribuiu com o delineamento e elaboração da pesquisa e a revisão do artigo.

\section{FONTES DE FINANCIAMENTO}

Faculdade Ceres (FACERES), São José do Rio Preto, São Paulo, Brasil.

\section{REFERENCIAS}

1. Farías-Antúnez $S$, Fassa $A G$. Prevalência e fatores associados à fragilidade em população idosa do Sul do Brasil, 2014. Epidemiol Serv Saúde. 2019;2(1):210-8.

2. Bernardi HLF, Motta LB. Desenvolvimento de aplicativo como ferramenta de apoio à investigação e prevenção de osteoporose. Rev Bras Geriatr Gerontol. 2018;21(4):420-30.

3. Baccaro LF, Conde DM, Costa-Paiva L, Pinto AM Neto. The epidemiology and management of postmenopausal osteoporosis: a viewpoint from Brazil. Clin Interv Aging. 2015;10(01):583-91.

4. Radominski SC, Bernardo W, Paula AP, Albergaria B, Moreira C, Fernandes CE, et al. Diretrizes brasileiras para o diagnóstico e tratamento da osteoporose em mulheres na pós-menopausa. Rev Bras Reumatol. 2017;57(S2):S452-S66.

5. Caires ELP, Bezerra MC, Junqueira AFTA, Fontenele SMA, Andrade CA, D'Alva CB. Tratamento da osteoporose pós-menopáusica: um algoritmo baseado na literatura para uso no sistema público de saúde. Rev Bras Reumatol. 2017;57(3):254-63.

6. Flores $\mathrm{CB}$. Prevalência de tratamento farmacológico para prevenção de fraturas ósseas em mulheres com osteoporose no município de Santa Maria - RS [dissertação]. Santa Maria: Universidade Federal de Santa Maria; 2015.

7. Cordeiro $\mathrm{CM}$. Tecnologia de prevenção secundária e tratamento da fratura de fêmur osteoporótica em mulheres na pós-menopausa no Brasil - análise de custo efetividade como ferramenta de gestão [tese]. Rio de Janeiro: Escola Nacional de Saúde Pública Sergio Arouca para obtenção do grau de Mestre; 2010.

8. Gomes LIC. Osteopenia e Osteoporose: factores modificáveis e não modificáveis [tese]. Porto: Universidade Fernando Pessoa; 2012.

9. Chen SJ, Chen YJ, Cheng CH, Hwang HF, Chen CY, Lin MR. Comparisons of different screening tools for identifying fracture/osteoporosis risk among community-dwelling older people. Medicine. 2016;95(20):1-10.

10. Hong-Mei Z, Hui-Ling L, Xuan W, Wei C, Dan C, Zhong-Zhi Z, et al. Clinical value of self-assessment risk of osteoporosis. Chin Med J. 2016;11(1):190-5. 
11. Cavalli L, Guazzini A, Cianferotti L, Parri S, Cavalli T, Metozzi A, et al. Prevalence of osteoporosis in the Italian population and main risk factors: results of BoneTour Campaign. BMC Musculoskelet Disord. 2016;17(01):396-7.

12. Jagarlamudi M, Umesh Rao V, Nitya K, Prathima P, Swati Rana A. Community based study on knowledge attitudes and practice of osteopororsis in women. Indo Am J Pharm Res. 2015;5(02):846-57.

13. lannetta R, Feitosa RB, lannetta O. Rastreamento das duas matrizes ósseas, proteica e inorgânica, em 192 sujeitos na adolescência. Estudo piloto coorte transversal. Prevençao primária da osteoporose. Rev Núcl Estudos Saúde Adolescent. 2015;12(2):51-8.

14. International Osteoporosis Foundation. Osteoporosis -patient brochure. Main strategies to take care of you bone after 50th [Internet]. 2013 [Acesso em 2019 Nov 30]. Disponível em: http://share.iofbonehealth.org/ WOD/2013/patient-brochure/PT/WOD13-patient_brochure-PT.pdf

15. Fernandes TRL, Oliveira JB, Lorencete TV, Amadei JL. Fatores associados à osteoporose em mulheres na pós-menopausa. Semina Cienc Biol Saúde. 2015;36(1):93-106.

16. Meira EC, Reis LA, Gonçalves LHT, Rodrigues VP, Philipp RR. Vivências de mulheres cuidadoras de pessoas idosas dependentes: orientação de gênero para o cuidado. Esc Anna Nery. 2017;21(2):e20170046

17. Andrade SAF. A Importância do Exame de Densitometria Óssea. Rev UNILUS Ensino Pesqui. 2016;3(30):11-7.

18. Silva ACV, Rosa MI, Fernandes B, Lumertz S, Diniz RM, Damiani MEFR. Fatores associados à osteopenia e osteoporose em mulheres submetidas à densitometria óssea. Rev Bras Reumatol. 2015;55(3):223-8.

19. Ministério da saúde (BR), Secretaria de Saúde. Portaria n 224, 26 de março 2014 [Internet]. 2014 [Acesso em 2019 Nov 30]. Disponível em: http://portalarquivos.saude.gov.br/images/pdf/2014/abril/02/ pcdtosteoporose-2014.pdf

20. Assumpção D, Domene SMA, Fisberg RM, Canesqui AM, Barros MBA. Diferenças entre homens e mulheres na qualidade da dieta: estudo de base populacional em Campinas, São Paulo. Ciênc Saúde Colet. 2017;22(2):347-58.

21. Pinto RL. Perfil Epidemiológico da ingestão de leite pela população de campinas: estudo de base populacional [dissertação]. Campinas: Universidade Estadual de Londrina; 2017.

22. Trindade MT, Bezerra NN, Starling OS, Viana ESM, Torres SAM, Gusman GS. Atenção farmacêutica na fitoterapia. Rev Cient Univiçosa. 2018;10(1):1074-80.

23. Pinheiro MM, Ciconelli RM, O'Jacques N, Genaro OS, Martini LA, Ferraz MB. O impacto da osteoporose no Brasil: dados regionais das fraturas em homens e mulheres adultos - The Brazilian Osteoporosis Study (BRAZOS). Rev Bras Reumatol. 2010;50(7):113-27.

24. Andrade PCO, Castro LS, Lambertucci MS, Lacerda MIPB, Araújo VPRJ, Bremm TAL, et al. Alimentação, fotoexposição e suplementação: influência nos níveis séricos de vitamina D. Rev Med Minas Gerais. 2015;25(3):432-7.

25. Bellan M, Pirisi M, Sainaghi PP. Osteoporose na artrite reumatoide: papel do sistema vitamina D/hormônio paratireóideo. Rev Bras Reumatol. 2015;55(3):256-63.

\section{Endereço para correspondência:}

Giulia Ohana Franco

Av. Anísio Haddad, 6751

Bairro: Jardim Morumbi

CEP: 5090-305 - São José do Rio Preto - SP - Brasil

E-mail: giuliaohanafranco@hotmail.com

Como citar: Franco GO, Sestini G, Dumbra GAC, Conte MS, Pacca FC, Roma DVP, et al. Ações de prevenção primária e secundária relacionadas aos fatores de risco para osteoporose. Rev Bras Promoç Saúde. 2020;33:9644. 